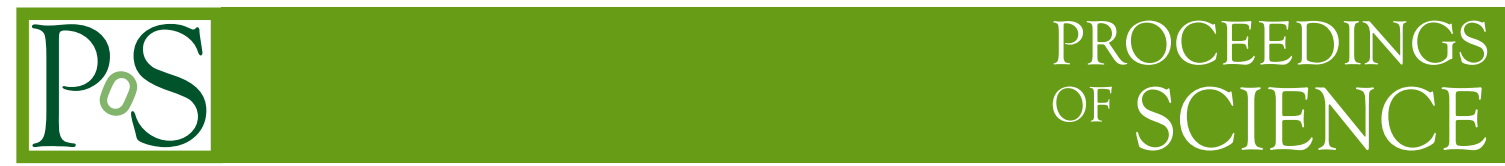

\title{
The most precise measurements of the Crab nebula inverse Compton spectral component
}

\section{Roberta Zanin*ث}

University of Barcelona, Spain

E-mail: robertazanin@gmail.com

\section{Oscar Blanch}

Institut de Fisica d'Altes Energies, Bellaterra, Spain

E-mail: blanch@ifae.es

\section{Ruben Lopez}

Institut de Fisica d'Altes Energies, Bellaterra, Spain

E-mail: rlopezdifae.es

\section{Diego F. Torres}

IECC, Bellaterra, Spain

E-mail: dtorres@ieec.es

\section{Dieter Horns}

University of Hamburg, Germany

E-mail: dhorns@desy.de

\section{Manuel Mayer}

Stokkolm, Sweeden

E-mail: manuel.meyer@fysik.su.se

\section{Jonathan Martin}

IEEC, Bellaterra, Spain E-mail: jonatanmrtn@gmail.com 


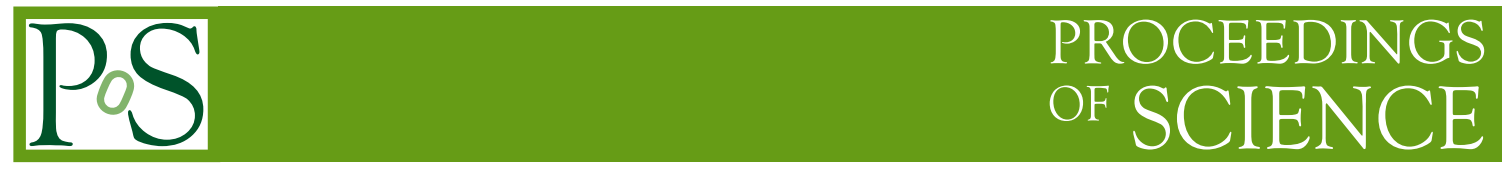

The Crab pulsar wind nebula (PWN) is one of the best studied astrophysical objects. Due to its brightness at all wavelengths, precise measurements are provided by different kind of instruments, allowing for many discoveries, later seen in other non-thermal sources, and a detailed examination of its physics. Most of the theoretical models for PWN emission are, in fact, based on Crab nebula measurements. The Crab nebula shows a broad-band spectrum spanning from radio frequencies up to VHE gamma rays and consists of two components, one of synchrotron origin and the other one due to radiative inverse Compton losses, starting at a few $\mathrm{GeV}$. We will report the most precise measurements of the inverse Compton component from the Crab Nebula by combining data by the Large Area Telescope (LAT) on board of the Fermi satellite (1-300 GeV) and by the stereoscopic MAGIC system $(>50 \mathrm{GeV})$. At low energies, the MAGIC results, combined with the Fermi/LAT data, show a flat and broad inverse Compton peak. The overall fit to the data between $1 \mathrm{GeV}$ and $30 \mathrm{TeV}$ is well-described by a modified log-parabola function with an exponent of 2.5 , and places the position of the inverse Compton peak at around $53 \mathrm{GeV}$. The spectral measurements obtained by the MAGIC collaboration cover more than three decades in energy, and could help to address the still-open question about the maximum energy reached by the parent electron population. The broadness of the inverse Compton peak cannot be reproduced by either the constant B-field model or the MHD flow model. The conclusion, based on earlier data, that simple models (constant Bfield, spherical symmetry) can account for the observed spectral shape has to revisited at the light of the new MAGIC results. On the other hand, the time-dependent 1D spectral model provides a good fit of the new VHE results when considering a $80 \mu \mathrm{G}$ magnetic field. However, it fails to match the data when including the morphology of the nebula at lower wavelengths.

The 34th International Cosmic Ray Conference,

30 July- 6 August, 2015

The Hague, The Netherlands

* Speaker.

${ }^{\dagger}$ A footnote may follow. 


\section{Introduction}

The pulsar wind nebula (PWN) associated with the Crab Pulsar is a leftover of a supernova explosion that occurred in 1054 and it is powered by the pulsar PSR B0531+21 at its center. The Crab Nebula continuously supplies relativistic particles, mainly positrons and electrons, that advect in the magnetized wind of the neutron star. These relativistic particles are thought to be accelerated to a power-law distribution either via a Fermi-like acceleration process taking place at the termination shock (TS) [1], or via shock-driven reconnection in a striped wind [2]. The down-stream flow interacts with the surrounding magnetic and photon fields creating the PWN. The nebula emits synchrotron radiation which is observed from radio frequencies up to soft $\gamma$ rays. This emission is well described by the magnetohydrodynamic (MHD) model of [3]. At higher energies (above $1 \mathrm{GeV}$ ), the overall emission is instead dominated by the Inverse Compton (IC) up-scattering of, mainly, synchrotron photons by the relativistic electrons in the nebula [4].

The Crab Nebula is one of the best studied objects in the sky. Due to its brightness at all wavelengths, precise measurements are provided by different kinds of instruments, allowing for many discoveries, later seen in other non-thermal sources, and a detailed examination of its physics. The IC emission from the Crab Nebula was detected for the first time above $700 \mathrm{GeV}$ by the pioneering Whipple imaging atmospheric Cherenkov telescope (IACT) in 1989 [5]. Since then, the imaging Cherenkov technique has been successfully used to extend the Crab Nebula differential energy spectrum from few hundred $\mathrm{GeV}$ up to $80 \mathrm{TeV}([6,7])$. However, the spectrum below $200 \mathrm{GeV}$ has been observed only recently, revealing the long-anticipated IC peak in the distribution. At low energies, space-based instruments, like Fermi-LAT, have improved the sensitivity in the energy range between few and hundred $\mathrm{GeV}$ [8]; whereas, on the other side, ground-based IACTs with larger reflective surface reached lower energy thresholds, below $100 \mathrm{GeV}$. The observations carried out by the stand-alone first MAGIC telescope (MAGIC-I) showed a hardening of the spectrum below a few hundred $\mathrm{GeV}$ [9]. However, in previous studies using MAGIC-I and Fermi-LAT measurements, the spectral overlap required to make a precision measurement of the IC peak energy was not achieved. Moreover, the quality of the available data around the IC peak was insufficient to rule out existing PWN models or at least distinguish between them. At energies above $\sim 10 \mathrm{TeV}$ there is a disagrement between HEGRA [6] and HESS [7] measurements. This may be due to systematic uncertainties of between the two instruments or may indicate a real spectral variability in the nebula, but it still remains an open question.

The goal of this work is to use the MAGIC stereoscopic system to measure, with high statistical precision, the Crab Nebula differential energy spectrum down to energies of $50 \mathrm{GeV}$, and, once combined with the Fermi-LAT results, to compare the overall spectral measurement with state-ofthe-art PWN models.

\section{Observation and analysis}

MAGIC currently consists of two $17 \mathrm{~m}$ diameter IACTs located in the Canary Island of La Palma (Spain) at a height of $2200 \mathrm{~m}$ above sea level. It is sensitive to very-high-energy (VHE) gamma rays in the energy range between a few tens of $\mathrm{GeV}$ and a few tens of TeV. MAGIC started operations in autumn 2004 as a single telescope, MAGIC-I, and became a stereoscopic system 
five years later in 2009. During the summers 2011 and 2012, MAGIC underwent a major upgrade involving the readout systems of both telescopes and the MAGIC-I camera [10].

To achieve the first goal of this work we used MAGIC stereoscopic observations of the Crab Nebula carried out between October 2009 and April 2011, before the above-mentioned upgrade. The instrument performance in this period is described in detail in [11]. The selected data set includes observations performed in wobble mode at zenith angles between $5^{\circ}$ and $62^{\circ}$, resulting, after data quality selection cuts, in 69 hrs of effective time.

The analysis was performed by using the tools of the standard MAGIC analysis software [13]. For the first considered data set we used the sum image cleaning, a new algorithm which lowers the analysis energy threshold to $55 \mathrm{GeV}$ and provides a $15 \%$ improvement in sensitivity below $150 \mathrm{GeV}$ [14]. More details on the analysis can be found in [15].

\section{The IC peak}

The spectral energy distribution (SED) obtained with the first data set of Crab nebula data, shown in Figure 1, covers almost three decades in energy, from $50 \mathrm{GeV}$ up to $30 \mathrm{TeV}$. It has five spectral points per energy decade and statistical errors as low as $5 \%$ below $150 \mathrm{GeV}$. Figure $1 \mathrm{com}$ pares the obtained results to the measurements by other IACTs as well as to the Fermi-LAT ones [16] (33 months of LAT data). The overall IC emission from the Crab Nebula is usually approximately described by a log-parabola function, and the corresponding fit to the MAGIC spectral points gives a flux normalization at $1 \mathrm{TeV}$ of $f_{0}=(3.23 \pm 0.03) 10^{-11} \mathrm{TeV}^{-1} \mathrm{~cm}^{-2} \mathrm{~s}^{-1}$, a photon index $\alpha=2.47 \pm 0.01$ and a curvature parameter $\beta=-0.24 \pm 0.01$ with a $\chi_{\text {red }}^{2}$ of 20/11. Among the other functions used in literature to describe this emission, the single power law fails in representing the data due to an obvious curvature in the measured spectrum, whereas the power law with exponential cutoff results in a flux normalization $f_{0}=(3.80 \pm 0.11) 10^{-11} \mathrm{TeV}^{-1} \mathrm{~cm}^{-2} \mathrm{~s}^{-1}$, a photon index $\alpha=2.21 \pm 0.02$, and an energy cutoff $E_{c}=6.0 \pm 0.6 \mathrm{TeV}$ with a $\chi_{\text {red }}^{2}$ of $35 / 11$. The overall systematic uncertainty affecting the measurement of the Crab Nebula SED includes three different classes of effects: one on the energy scale at the level of $15-17 \%$, the second in the flux normalization about $11 \%$, and the third one on the spectral shape which can be expressed in terms of $\alpha \pm 0.03_{\text {sys }}$ and of $\beta \pm 0.05_{\text {sys }}$ [15].

To ensure independence from theoretical modeling, and assuming that the easiest approximation for the IC contribution of the Crab Nebula emission is a log-parabolic shape, we estimate the position of the IC peak by a log-parabola fit to the data. The most robust fit is obtained by considering MAGIC and Fermi-LAT spectral data together since they cover both sides of the IC peak. We, therefore, perform a joint fit to the MAGIC and Fermi-LAT spectral data points starting from $1 \mathrm{GeV}$, corresponding to the energy of the lowest spectral point of the Fermi-LAT spectrum where the IC contribution dominates over synchrotron emission. The fit results in an IC peak position, $\mathrm{E}_{I C}$, at $(53 \pm 3) \mathrm{GeV}$. The investigation on how the systematic uncertainties may alter the fit result (treated in detail in [15]) yields to an IC peak position of $\left(53 \pm 3_{\text {stat }}+31_{\text {sys }}-13_{\text {sys }}\right) \mathrm{GeV}$.

Since we see a rather flat IC peak in the data, we also looked for a spectral model which better reproduces the new observational results from $1 \mathrm{GeV}$ to $\sim 30 \mathrm{TeV}$. To improve the likelihood of the fit, we considered functions with extra free parameters, and the most satisfactory one was the following modified log-parabola function: $E^{2} \times d N / d E=10^{\log f 0+C\left(\log \frac{E}{E_{I C}}\right)^{a}}$. Such a fit function, 
with one more free parameter a than a log-parabola discussed above, provides acceptable results with a $\chi_{\text {red }}^{2}=35 / 26$, locating the IC peak at $\mathrm{E}_{I C}=(48 \pm 2) \mathrm{GeV}$. The resulting exponent a $=2.5$ \pm 0.1 produces a flatter peak than the one obtained by the canonical quadratic function. Even though the fit function provides a good fit to the joint data set without any shift in energy scale or flux normalization, it is not physically motivated. The resulting peak position remains within the uncertainty quoted earlier: $\left(53 \pm 3_{\text {stat }}+31_{\text {sys }}-13_{\text {sys }}\right) \mathrm{GeV}$. The main result of this study is that the IC peak is flatter and broader than expected and this has important implications at the theoretical level, as shown in the following subsection.

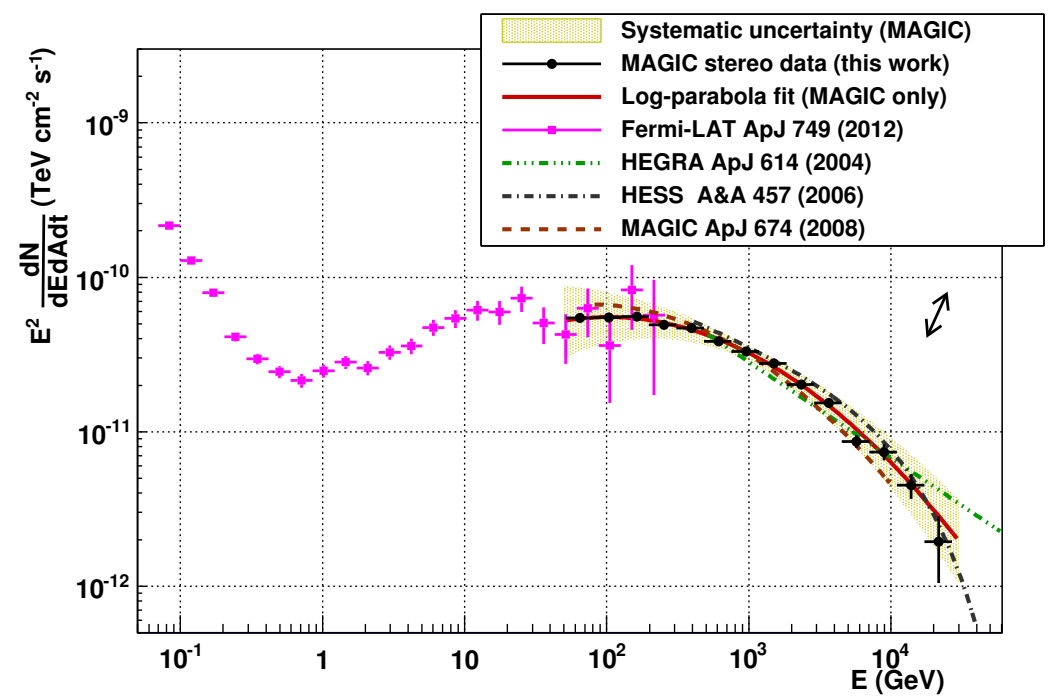

Figure 1: SED of the Crab Nebula from $100 \mathrm{MeV}$ to $\sim 30 \mathrm{TeV}$ obtained by Fermi-LAT and MAGIC together with the fit results from other $\gamma$-ray experiments. The black arrow indicates the systematic uncertainty on the energy scale, whereas the shaded area indicates the systematic uncertainty on the flux normalization and the photon index.

\subsection{Discussion}

The broad-band SED of the Crab Nebula has been tested against three models:

- The magneto-hydro-dynamic (MHD) flow model [17].

It is based on the analytical modelization of the structure of the downstream pulsar wind for the simplified case of a spherical symmetric ideal MHD flow, as in [3]. While this model is a good representation of the synchrotron emission, it totally fails in reproducing the IC component, indicating that the spatial structure of the magnetic field is not consistent with the data.

- The Static, Constant B-field model was introduced in [17] and follows the prescription put forward in [21]. The Crab Nebula is assumed to be homogeneously filled with a constant magnetic field and two distinct electron populations: relic electrons (responsible for the radio synchrotron emission) and wind electrons. In the IC emission region, the data is poorly 
described and the model only converges if an ad-hoc (unrealistically large) systematic uncertainty of $17 \%$ is assumed, resulting in $\chi_{\text {red }}^{2}=1.57$. The higher quality (i.e. smaller error bars) of the Fermi-LAT data together with the MAGIC data shows a rather flat peak now, which cannot be reproduced in the model. If we repeated the exact procedure from the 2010 paper but only using the updated Fermi-LAT data, we would find a lower B-field and the model would undershoot the MAGIC data at almost all energies. We, therefore, conclude that the constant B-field model cannot reproduce the flat peak of the IC SED. For energies above the peak, the predicted spectrum is too soft with too little curvature as compared to the new MAGIC data.

- The time-dependent 1-dimension model $[18,19,20]$. Such model solves the diffusionloss equation numerically devoid of any approximation, considering synchrotron, IC and Bremsstrahlung energy losses. It can satisfactorily reproduce the VHE data up to few $\mathrm{TeV}$ under the assumptions of a low magnetic field of less than hundred IijG. It fails, however, to provide a good fit of the new spectral data if the observed morphology of the nebula (smaller size at shorter wavelengths, as in [21]) is adopted.

We conclude that more theoretical work on the Crab Nebula modeling must be done to simultaneously fit the observed morphology and the spectral energy distribution. The broad-band IC spectrum is in principle sensitive to the spatial structure of the magnetic field and hence can be used for future models.

\section{References}

[1] Arons, J., Tavani, M., ApJ, 1994 90:797

[2] Sironi, L., Spitkovsky, A., ApJ, 2011, 741:39

[3] Kennel, Coroniti, ApJ, 1984, 283: 710

[4] Atoyan, A.M., Aharonian, F., MNRAS, 1996, 278: 525

[5] Weekes, T. et al, ApJ, 1989, 342:379

[6] Aharonian, F. et al, ApJ, 2004, 614: 897

[7] Aharonian, F. et al, A\&A, 2006, 457: 899

[8] Abdo, A. et al., ApJ, 2010, 708:1254

[9] Albert, J. et al., ApJ, 2008, 674:1037

[10] Aleksić, J. et al, Astropart., 2015,

[11] Aleksić, J. et al, Astropart., 2012 35: 435

[12] Aleksić, J. et al, Astropart., 2015,

[13] Zanin, R. et al, ICRC proceedings, 2013

[14] Lombardi, S. et al, ICRC proceedings, 2011

[15] Aleksić, J. et al, JHEAp, 2015,

[16] Buehler, R. et al, ApJ, 2012 749:26 
[17] Meyer, M. et al, A\&A, 2010, 523:A2

[18] Martin, J. et al, ApJ, MNRAS, 2012 427:415

[19] Torres, D. et al, ApJ Letters, 2013a 763:L4

[20] Torres, D. et al, MNRAS, 2013b 436:3112

[21] Hillas, et al, ApJ, 1998 503:744 